\title{
Cost analysis of on-premise versus cloud-based implementation of Moodle in Kufa University during the pandemic
}

\author{
Abdulhussein Abdulmohson ${ }^{1}$, Mohammed Falih Kadhim ${ }^{2}$, Othman M. Hussein Anssari², \\ Ahmed A Al-Jobouri' ${ }^{2}$ \\ ${ }^{1}$ Department of Computer Science, College of education, University of Kufa, Najaf, Iraq \\ ${ }^{2}$ Information Technology Research and Development Center, University of Kufa, Najaf, Iraq
}

\begin{tabular}{l} 
Article Info \\
\hline Article history: \\
Received Apr 12, 202 \\
Revised Dec 23, 202 \\
Accepted Jan 19, 2022 \\
\hline Keywords: \\
Cloud computing \\
Coronavirus \\
Datacenter \\
E-learning \\
Kufa university
\end{tabular}

\begin{abstract}
Many organizations often use physical hardware resources, such as storage devices and firewall, to store their essential applications and data. Recently, the Coronavirus pandemic represented a significant challenge for the University of Kufa, which utilize an on-premise data center for e-learning since 2009. Whether the learning management system (LMS) is installed on an on-premise data center or the cloud, it is crucial for any university, to decide which implementation is more suitable because of the differences between the two options, especially in terms of cost. This study uses the total cost of ownership (TCO) model to highlight the cost aspect when using onpremise datacenter versus cloud-based implementation for e-learning and to determine which option is cost effective. The results may help other universities, inside or outside Iraq, deciding which implementation is more suitable (financially) for the organization. The final results show that the cloud-based solution costs approximately $20 \%$ less than the currently used on-premise option. Despite all drawbacks of on-premise datacenter such as unstable electricity, bad Internet service, and costing more than cloud hosting, it maybe still more convenient in the case of the University of Kufa due to the sensitive data stored in the data center.
\end{abstract}

This is an open access article under the CC BY-SA license.

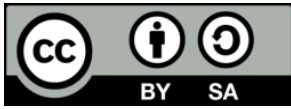

\section{Corresponding Author:}

Abdulhussein Abdulmohson

Department of Computer Science, College of education, University of Kufa

Najaf, Iraq

Email: abdulhussein.fadhel@uokufa.edu.iq

\section{INTRODUCTION}

In the end of 2019, the world has witnessed a new epidemic affecting the whole world and led to the suspension of traditional education in $60 \%$ of the world's institutions [1]. In Iraq, the virus appeared in March 2020. Soon after, the Iraqi Ministry of Higher Education decided to stop traditional education and shift towards E-learning [2], [3]. The University of Kufa, which was chosen as a case study for this research, took advantage of a data center that was established in 2009 utilizing Moodle as a learning management system (LMS) 21 colleges serving more than 29,193 undergraduate and 1,911 postgraduate students, and 2,148 faculty members [4]. E-learning at the beginning of its use in 2013 was not effective, rather it was used to a very limited extent, therefore the administration was carried out by only one server. Kufa university data center initially contained 9 servers, one of them only for e-learning and the rest for managing and distributing university services [5]. With the adoption of e-learning at the University of Kufa after the pandemic, the university network administration officials had to carry out a major upgrade plan to handle the increased load. As shown in Figure 1 which compare the network infrastructure before and after the upgrade, the plan is to expand the data center to 9 servers used exclusively for e-learning across 21 faculties (each faculty has a 
separate site) in addition to 8 servers for managing and distributing university services, some intranet services while other over internet. Moodle LMS is installed and used on all servers across the university.

Moodle is a free learning platform used as a learning management system. The platform was created by Martin Dougiamas to help academic staff manage courses in an online environment. The platform is managed and provided by an Australian company called Moodle HQ, which is made up of 50 developers and is financially backed by a network of eighty-four [6]. The use of e-learning and the Moodle platform requires internet to access the university data center. The problem of the internet in Iraq is one of the fundamental problems that have never been resolved. The problem is due to poor infrastructure, lack of resources to upgrade, and lack of incentives and encouragement from the government [7], [8]. Additionally, the cost of internet service is significantly high compared to neighboring countries, where the price of one mega in Iraq is 54 dollars, while it is less than a dollar in neighboring countries [9].
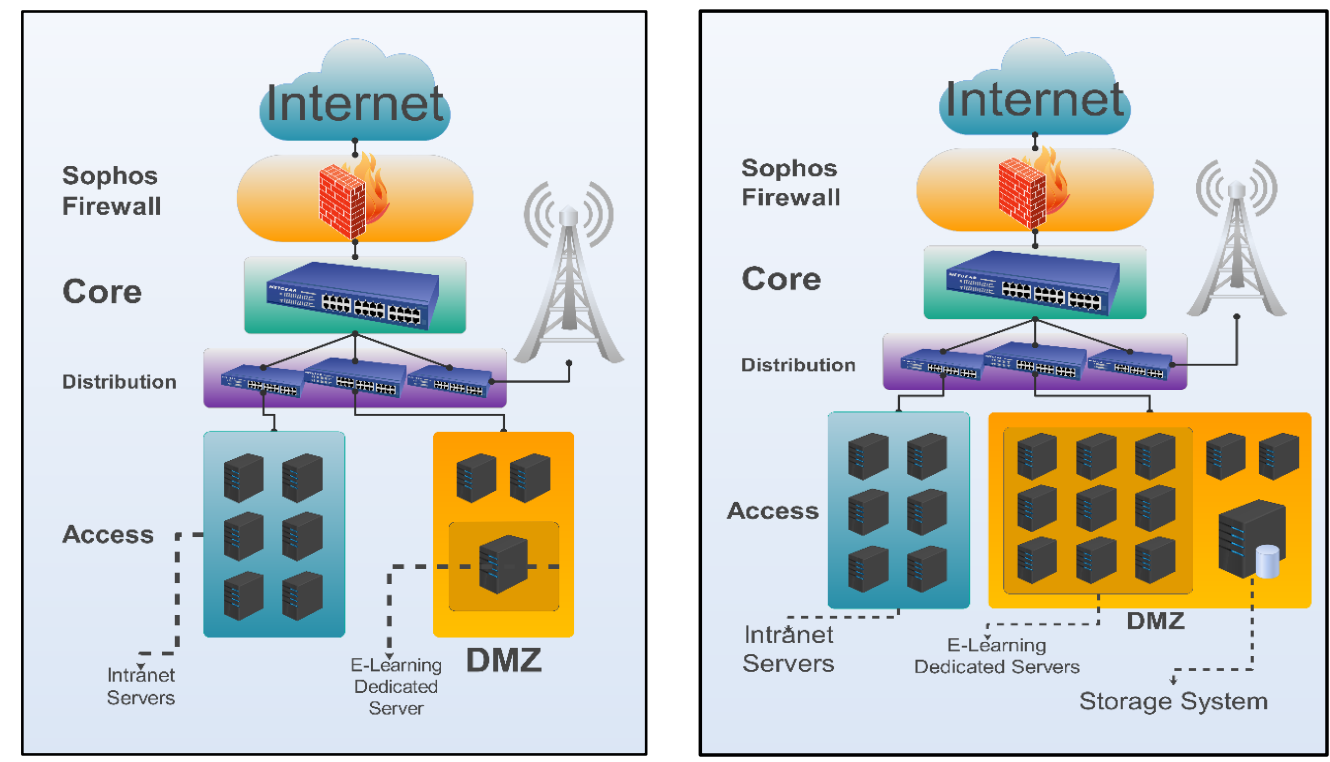

Figure. 1. Network topology before and after upgrade

The internet connectivity in e-learning is considered the most common problem for both students and academic staff. In addition to the problems with the Internet service, electricity is another major problem that all people in Iraq are suffering from. The governments have been unable to find solutions to that problem since 2003, as this sector has suffered from wars, neglect and sabotage. The Iraqi knowledge center classified this service as the worst service provided in Iraq [10], [11].

This study may not be the first in this area but it has two contributions that differ from other studies in this area, firstly it critically analyzes the on-premise data center since it is already established, in contrast, most studies focus by comparison on building a data center from scratch or moving toward cloud computing. Secondly, the conditions in which the data center operate is an exceptional circumstance due to Corona pandemic, as the impact of this epidemic on the use of the universities data center as a result of the shift from traditional education to electronic education, which required intensive use of data center resources, especially during electronic exams.

\section{RELATED STUDIES}

On-premise versus cloud-based implementation of LMS, in any educational institution, before implementing a learning management system, the first step is deciding how to deploy the system. Whether it is on-premise or cloud-hosted, the cost of building and maintaining LMS can be significant which may hinder deploying the system especially in Iraq due to continuous financial crisis. Generally speaking, onpremise LMS is hosted locally on servers and requires hardware and software in addition to human resources to build and maintain the on-site network to host the LMS. Cloud-based LMS, on the other hand, the hardware and software equipment are delivered as a service via the internet which can be purchased by the staff in the form of monthly or annual subscription [12]. 
Studies directly comparing the two architectures in term of cost are limited. However, many research papers outlined cons and pros for each architecture in terms of accessibility, installation process, and customizability [13]. Highlighted key differences between the two models. According to the author, onpremise LMS is hosted locally and require software to be installed on the server unlike cloud-based solution which is remotely accessed and service providers are responsible for providing the infrastructure that hosts the LMS [13]. The on-premise LMS installation process typically requires more time and effort but it provides wide range of customization choices that the cloud-based does not have. Service wise, cloud-based LMS usually provided as subscription model such as Software as a Service (SaaS) which is also the most widely used computing service compared to other cloud-based models like IaaS and PaaS [14], [15]. The main benefit of cloud LMS solutions, specifically SaaS, is scalability which means service automatically scales based on usage unlike on-premise solution which requires upgrading hardware unites to increase capacity and performance [16], [13]. As a result, cloud-based service offers better resource manageability without requiring technical human resources [16], [17].

As mentioned, few literatures compared between on-premise and cloud-based implementation of LMS. Admits that cloud-based LMS can reduce the number of technical man power required to maintain LMS, however it can still be more expensive than the locally hosted LMS [16]. In contrast, [18]-[21] believe that on-premise LMS can cost substantially more due to requirement of network hardware, software and specialized technical staff to manage and maintain the system. Furthermore, on-premise solution requires electric power and cooling equipment which is typically expensive. A study by [19] calculated cost of implementing LMS using both cloud-based and on-premise solutions. The author used total cost of ownership (TCO) approach to determine the real cost. Since there is a wide range of available cloud solutions, the author compared total cost across multiple SaaS providers namely Amazon Cloud, Google Cloud, and HP cloud. The final result shows that hosting LMS on Google cloud is significantly cheaper compared to on-premise hosting [19], [22]. The difference in cost is mainly due to ICT infrastructure requirements and staff salaries.

Another reason why some institutions implemented cloud-based LMS is reliability [21]. As mentioned above, cloud services are highly scalable and dynamic. Subscription level is instantly changing depending on demand. Which is why cloud solution is significantly more reliable compared to the mostly fixed specifications of the on-premise solution [23].

Some studies highlighted another important aspect of LMS which is network security which is one of the main reasons some universities implemented on-premise LMS. On-premise hosting keeps student's data private and secure within the university network [18], [19]. Privacy concerns and legislative rules are among the reasons why some universities rejected cloud implementation of LMS [24]. Due to the pandemic crisis, $60 \%$ of academic institutions has temporarily suspended the traditional education technique [1]. Currently in Iraq, almost all university has shifted toward online LMS. In order to implement LMS in any educational organization, it is important to decide whether the system is installed on an on-premise data center or on the cloud. Unfortunately, no research has addressed the cost aspect of the two options when Moodle is used as an LMS. Furthermore, due to continuous financial crisis in Iraq, it important for educational organizations to understand which option is cost-effective. This study highlights the cost aspect when using on-premise datacenter versus cloud-based implementation for e-learning. The total cost of ownership (TCO) model is used in the calculation process which is discussed in the following section.

\section{METHODOLOGY}

This paper focuses on the cost aspect when implementing e-learning using on-premise data center in comparison with cloud-based solution. The total cost of ownership (TCO) model was used in the calculations, which in our case takes into account the costs of the Internet, electricity and the devices obsolete. The calculations do not take into account the costs of the data center itself, as it was already in place before the pandemic, nor financing the IT staff because they are originally on permanent contract and receive monthly payment before and after the pandemic.

\subsection{Electricity power}

In any data center, it is important to ensure consistent power supply 2417 . As mentioned earlier the entire country suffers from bad electricity service and as the number of servers increased, the administration had to develop alternative power source in the event of a power failure. One of the alternatives is using electrical generation system powered by solar energy. The gas generator was primarily used for many years to supply the electricity in addition to the grid power line. But the generators were not enough to ensure continuous operation because of some recurring problems, such as running out of fuel and the lack of materials which are necessary for the periodic maintenance work, in addition to problems of operating the generator after the official working hours. 
The administrator of datacenter has adopted the idea of supplying clean electrical energy since 2006, and this was achieved by installing the first solar system in 2008. Ten solar cells with a capacity of 100-watt solar cells have been installed. The system included eighteenth batteries and two inverters in order to equip the servers' room with about twenty amp at a cost of more than $\$ 15000$ at that time.

Later on, the solar energy system has been updated by adding twenty-eight solar panels with a capacity of 370 watts per panel with thirty batteries (each battery is a 200-amp gel-type deep cycle) at a cost of more than $\$ 13500$. The diagram below shows the entire energy system that powers the data center in the University of Kufa. Power consumption for each year after measuring total consumption for data center $\$ 3521$ year. It should be noted that electricity consumption tariffs are reduced by the Iraqi government towards universities and government institutions. The entire energy infrastructure is shown in Figure 2 showing the main energy sources that feed the data center.

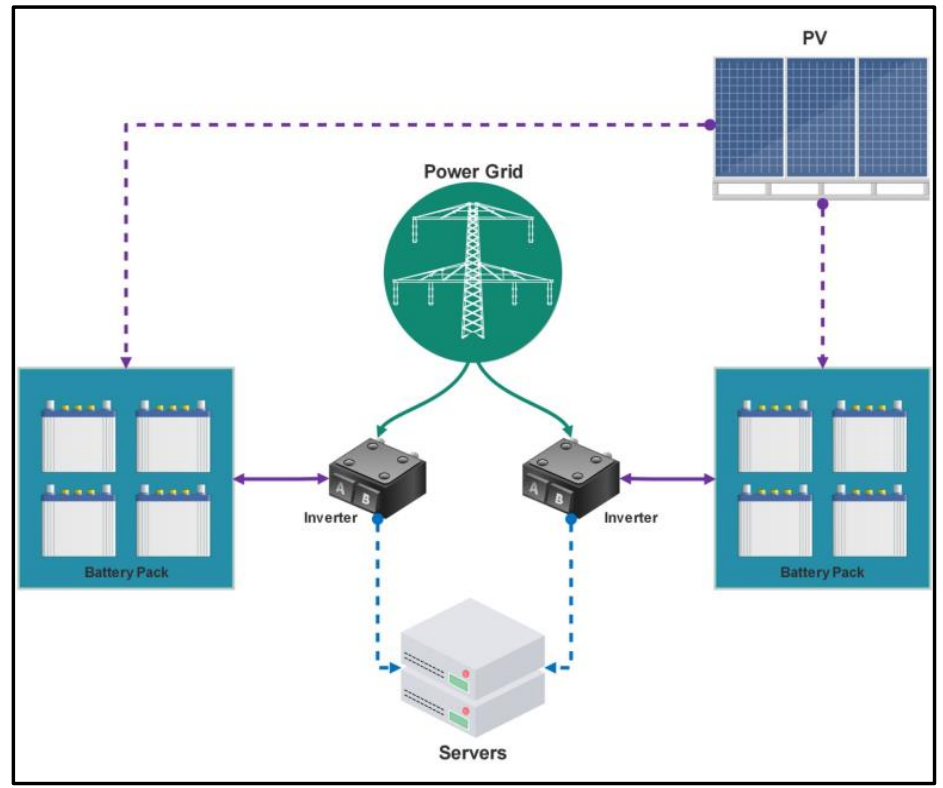

Figure. 2. Data center energy sources

\subsection{The cost of the internet service}

The cost of internet service in Iraq is relatively high in comparison to most neighboring countries. The University of Kufa uses $73 \mathrm{Mbps}$ bandwidth internet connection for use during the official working hours (from $8 \mathrm{AM}$ to $3 \mathrm{PM}$ ) and $8 \mathrm{Mbps}$ outside of working hours at a total cost of $\$ 7000 /$ month. This bandwidth is no longer sufficient after the outbreak of the epidemic as the use of e-learning has increased which required increasing the Internet package to $140 \mathrm{Mbps}$ during the official working hours and $60 \mathrm{Mbps}$ outside the working hours. This has increased the total cost to $\$ 13400 /$ month.

\subsection{Devices obsolete}

One of the most typical problems facing data center administrator is device obsolete where the devices lose compatibility with the recent programs in addition to the discontinuation of support provided by the manufacturers. In these cases, a newer technology should be sought. The life cycle of devices, especially servers, is usually from 4-5 years [25]. Despite the fact that these devices will continue to operate even after reaching end of life, continue to use it is highly risky as it may lead to losing students data as these systems no longer receive security updates. Unfortunately, many organizations try to keep their devices for as long as possible. And when the devices stop receiving updates from the manufacturer, the performance of the device will decrease and eventually unable to keep up with the demand, which leads to the necessity of updating the equipment or buying new devices, which are often very expensive. In the case of University of Kufa, and due to the lack of financial support, most devices work for at least 10 years before they are replaced. Table 1 shows estimated costs for the essential datacenter equipment. Full details have not been provided due to security reasons. In brief, the approximate cost of equipment obsolescence will be $10 \%$ of the total cost and equal to $\$ 39950$. 
Table 1 Cost of datacenter equipment

\begin{tabular}{lcc}
\hline Devices & Number of devices & Estimate total cost \\
\hline Servers & 11 & $24200 \$$ \\
Switch & 4 & 2000 \\
Cables & & 250 \\
Solar system & & 13500 \\
\hline
\end{tabular}

\subsection{Firewall cost}

Data center administrator use Sophus as a firewall and internet distributer system. The system is used to protect the network, internally and externally, from targets that affect the network. Sophus detects hidden user, app, and network threats and is unique in its ability to automatically respond to security incidents by isolating compromised systems with the security heartbeat. We will not go into the details of the firewall for security reasons. The cost of Annual subscription renewal for this firewall system is $\$ 4500$.

\subsection{Cloud based architecture}

Switching to cloud-based architecture means the data center at the University of Kufa will only provide services related to Internet distribution, student registration, electronic technical support, test systems for educational courses and others while all e-learning related services will be transferred to the cloud. The cloud-based system with the specifications shown in Table 2 should be able to serve 8000 concurrent users and 21 colleges without problems. Figure 3 shows typical users of cloud-based infrastructure. Primarily, the students, academics, and employees are considered the users, while technicians represent those who manage and administrate the services provided by the cloud environment.

Figure 4 illustrates the architecture of cloud-based learning management system utilizing three main servers. The idea is to turn all these individual servers into a cluster in which the workload is equally distributed on each server using load balancing technology. This technology can optimize hardware utilization and avoid overloading a single server when the other servers are idle.

Table 2. Moodle cloud specification

\begin{tabular}{lcl}
\hline \multicolumn{1}{c}{ Function } & Qty & \multicolumn{1}{c}{ Description } \\
\hline Moodle App server & 3 & (32 vCPU, 64GB RAM) \\
Moodle App server storage & 3 & 256 GB \\
Database & 1 & Compute Gen 5 (64 vCPUs, 320 GB RAM) \\
Database storage & 1 & 1TB \\
Database backup & 1 & 1TB \\
Moodle data Storage & 1 & 2TB (NetApp Files) \\
Load balancer & 1 & 5 Load Balancer rules + 1000GB Data processed \\
Bandwidth & 1 & 2TB Outbound Data Transfer \\
\hline
\end{tabular}

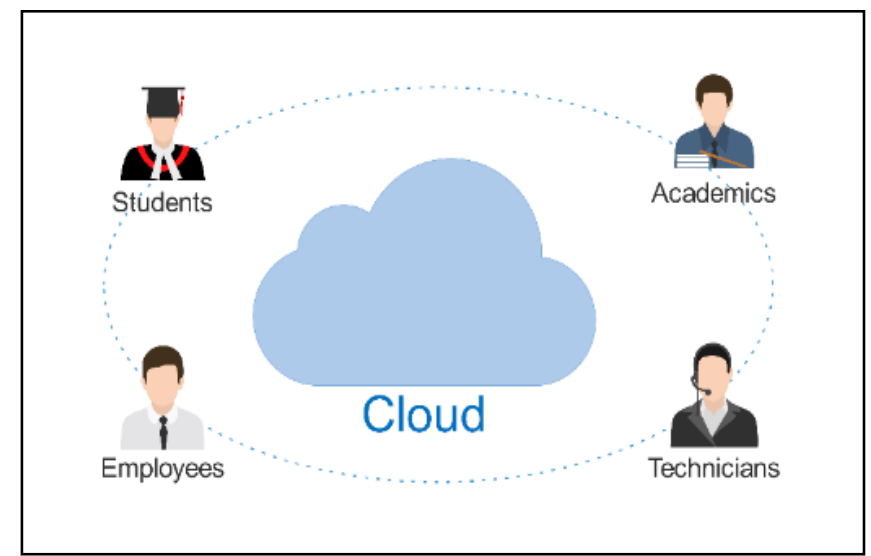

Figure 3. Cloud based architecture 


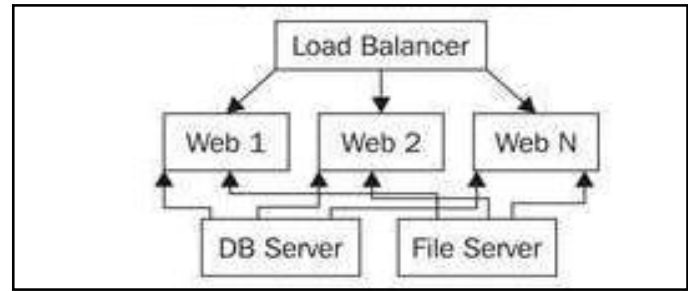

Figure 4. Architecture of cloud-based solution

Typically, these servers run Linux in addition to Apache, MySQL, and Perl which usually referred as LAMP for short. PHP is used as the main scripting language in which its' components is sits inside the Apache server while SQL is for database management. The Figure 5 shows the main components of Moodle system Moodle-cloud is superb when it comes to backing up the data stored on the servers. The system provides multi-stage backup mechanism where all data are simultaneously and seamlessly stored on multiple servers to ensure ability to retrieve data when a failure occurs.

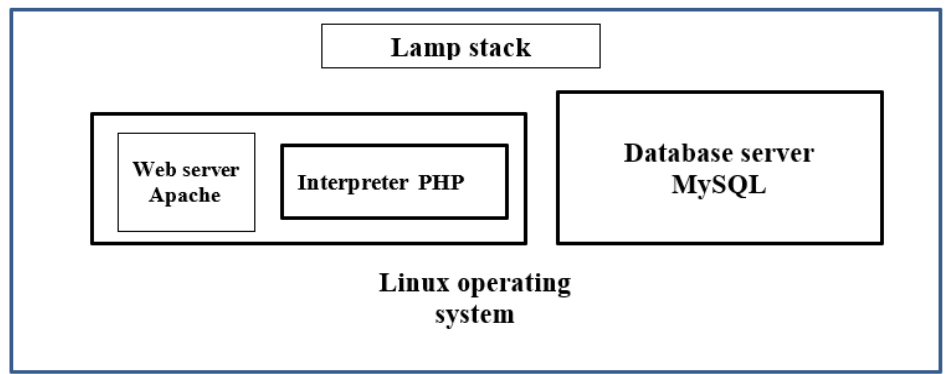

Figure 5. Components of Moodle system software

Advantages of Moodle-cloud: The Moodle-cloud has three advantages over the on-premise based solution

- Cost effective: Comparing TCO of both solutions, which is explained in details in later sections, shows that Moodle-cloud is more cost effective.

- Scalable: Moodle-cloud provides high availability solution as the cloud scale dynamically to meet the increased demand without having to physically upgrade server hardware which is the case with onpremise solution.

- Hassle-free: No need to deal with updates and periodic maintenance which makes Moodle-cloud trouble free experience.

\section{RESULTS}

\subsection{Analyzing the total cost}

The costs of using cloud-based environment are summarized in the offer submitted by one of the companies specialized in providing e-learning service through the Moodle platform on Azure cloud.

Hosting Servers (Azure Microsoft) $=10,128$ USD/Month

LAMP bundle including the following services: backup, server configuration, testing, installing SSL certificate on all servers, upgrading, installing monitoring solution. All these services cost $\$ 15000$ (one time). In summary, total cost $=10128 * 12+15000=\$ 136536$

It is certain that the use of this offer will help eliminate problems related to the supply of electrical power and the weakness of the Internet signal in addition to technical problems related to maintaining the software and other security problems as the hosting company is in charge of protecting the data. On the other hand, the high cost of this solution is a significant drawback.

\subsection{On-premise total cost}

The annual cost of on-premise solution is explained below. Again, it is important to note that the cost excludes IT staff expenses as they are on permanent contract with the government and receive monthly wages. 
- Power consumption $=\$ 3520$

- Internet: $\$ 13400 * 12=\$ 160800$

- Devices obsolete: $\$ 3950$

- Firewall: \$4500

- Total: $172750 /$ year

\section{CONCLUSION}

TCO analysis shows that the total cost of using cloud-based LMS is about $20 \%$ less compared to the cost of using the current on-premise data center. Even though it costs less, the currently implemented datacenter is still more convenient for a number of reasons. As discussed earlier the datacenter at the University of Kufa does have more purposes than e-learning. Services like managing internet accounts for staff, hosting Intranet applications, student registration system, managing student accounts, and other locally hosted services are all managed and stored locally within the datacenter space. If these services are required to be completely moved to the cloud, the subscription cost would substantially increase. Not to mention the fact that most of these data are sensitive data such as students' marks, accounts, employee's personal data, and the university digital mail. Having these data stored somewhere on the cloud may raise security concerns as these cloud networks are more subject to security breach. Additionally, using cloud hosting means the ultimate control and management is outside the institution which again raise concerns as the institution security protocols will not be applicable. Considering that the pandemic may end some day in the future, then the high demand of e-learning will definitely be reduced and thus switching to cloud computing will not be required as the on-premise data center will be more than enough for the task. Admittedly, it might be more feasible to use cloud hosting in the case of no data center was previously implemented in the university as building a datacenter from the ground up would cost too much.

\section{REFERENCES}

[1] B. M. Hashim, S. K. Al-Naseri, A. A.-Maliki, and N. Al-Ansari, "Impact of COVID-19 lockdown on NO2, O3, PM2. 5 and PM10 concentrations and assessing air quality changes in Baghdad, Iraq," Science of the Total Environment, vol. 754, 2020, doi: 10.1016/j.scitotenv.2020.141978.

[2] A. H. Mousa, S. H. Mousa, S. H. Mousa, and H. A. Obaid, "Advance acceptance status model for E-learning based on university academics and students," IOP Conference Series: Materials Science and Engineering, vol. 671, no. 1, 2020.

[3] Elameer and S. Amer, "Iraqi higher education digitizing trail during covid19 pandemic," European Journal of Molecular \& Clinical Medicine, vol. 8, no. 2, pp. 1231-1332, 2021

[4] University of Kufa, "Welcome to the University of Kufa," 2019. Accessed: Jan 19, 2022. [Online]. Available: https://uokufa.edu.iq/welcome-to-the-university-of-kufa?lang=en

[5] A. J. M. Karkar, H. K. Fatlawi, and A. A. Al-Jobouri, "Highlighting E-learning Adoption Challenges using data Analysis Techniques: University of Kufa as a Case Study," Electronic Journal of e-Learning, vol. 18, no. 2, pp. 136-149, 2020, doi: 10.34190/EJEL.20.18.2.003.

[6] Moodle. 2020. Accessed: Jan 19, 2022 .[Online]. Available: https://docs.moodle.org/310/en/History

[7] A. A.-Azawei, P. Parslow, and K. Lundqvist, "Barriers and opportunities of e-learning implementation in Iraq: A case of public universities," The International Review of Research in Open and Distributed Learning, vol. 17, no. 5, 2016, doi: 10.19173/irrodl.v17i5.2501.

[8] F. H. Al-Hammadany and A. Heshmati, "Determinants of Internet use in Iraq," International Journal of Communication, vol. 5, 2011.

[9] Iraq Energy, Iraq Business Perspectives (Part 1): Internet Infrastructure \& Stock Market Modernisation,” 2019. Accessed: Jan 19, 2022. [Online]. Available: https://iraqenergy.org/2019/06/09/business-perspectives-part1-internet-infrastructure-iraq-stockmarket-modernisation

[10] S. Rashid, Electricity problem in Iraq, Hamburg, 2012.

[11] H. H. Istepanian, "Iraq's electricity crisis," The Electricity Journal, vol. 27, no. 4, pp. 51-69, 2014, doi: 10.1016/j.tej.2014.04.006.

[12] N. Kshetri, "Cloud Computing in the Global South: drivers, effects and policy measures," Third World Quarterly, vol. 32, no. 6 , pp. 997-1014, 2011, doi: 10.1080/01436597.2011.586225.

[13] U. Kalsom, P. Hushalictmy, and K. Kiranjeet, "A conceptual study on using cloud based lms in higher education institutions in Malaysia," International Journal of Education and Pedagogy, vol. 1, no. 2, pp. 97-107, 2019.

[14] P. Rajasekar and P. Yogesh, "Scheduling multiple scientific workflows using containers on IaaS cloud," Journal of Ambient Intelligence and Humanized Computing, vol. 12, pp. 7621-7636, 2021.

[15] W.-L. Tsai, "Constructing assessment indicators for enterprises employing cloud IaaS," Asia Pacific Management Review, vol. 26, no. 1, pp. 23-29, 2021, doi: 10.1016/j.apmrv.2020.06.001.

[16] M. A. Seropian, G. R. KeelerViren, and N. Naik, "Comprehensive Healthcare Simulation: Program \& Center Development," Springer International Publishing, 2020.

[17] J. Nath and T. D. S. Shaw, "A cloud-based approach to library management solution for college libraries," Information Discovery and Delivery, 2020, doi: 10.1108/IDD-10-2019-0076.

[18] I. Bouchrika, Nouzha Harrati, Z. Mahfouf, and N. Gasmallah, "Evaluating the acceptance of e-learning systems via subjective and objective data analysis," Software data engineering for network elearning environments. Springer, Cham., pp. 199-219, 2018.

[19] J. Samson and R. R. Mtebe, "eLearning cost analysis of on-premise versus cloud-hosted implementation in Sub-Saharan countries," The African Journal of Information Systems, vol. 6, no. 2, p. 2, 2014

[20] R. Ferdiana, "The comparison of consumer cloud storage for a storage extension on the e-learning," 2016 6th International Annual Engineering Seminar (InAES). IEEE, 2016, doi: 10.1109/INAES.2016.7821930. 
[21] A. Gaur and M. Manuja, "Implementation framework for cloud based education-as-a-service," 2014 IEEE International Conference on MOOC, Innovation and Technology in Education (MITE), 2014, doi: 10.1109/MITE.2014.7020241.

[22] D. G. Chandra and M. D. Borah, "Cost benefit analysis of cloud computing in education," 2012 International Conference on Computing, Communication and Applications, 2012, pp. 1-6, doi: 10.1109/ICCCA.2012.6179142.

[23] M. Ketel, "Cloud services for e-learning," Proceedings of the 2014 ACM Southeast Regional Conference, 2014, doi: $10.1145 / 2638404.2638504$.

[24] S. A. Mokhtar, S. H. S. Ali, A. Alsharafi, and A. H. Aborujilah, "Cloud computing in academic institutions," Proceedings of the 7th international Conference on Ubiquitous information Management and Communication, 2013, doi: 10.1145/2448556.2448558.

[25] Beth Whitehead, D. Andrews, A. Shah, and G. Maidment, "Assessing the environmental impact of data centres part 2: Building environmental assessment methods and life cycle assessment," Building and Environment, vol. 93, pp. 395-405, 2015, doi: 10.1016/j.buildenv.2014.08.015.

\section{BIOGRAPHIES OF AUTHORS}

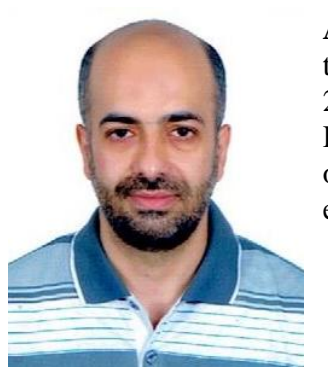

Abdulhussein Abdulmohson (iD 81 SC P He was born in Al-Najaf, Iraq in 1979. He obtained the B. Tech. Degree in Computer Engineering from University of Technology, Baghdad, Iraq, 2002. M.Tech. Degree in computer science and engineering from Osmania University, Hyderabad, India Hyderabad, India. He is a lecturer in the faculty of education at the University of Kufa, Najaf, Iraq where he has been a faculty member since 2015 . He can be contacted at email: abdulhussein.fadhel@uokufa.edu.iq.

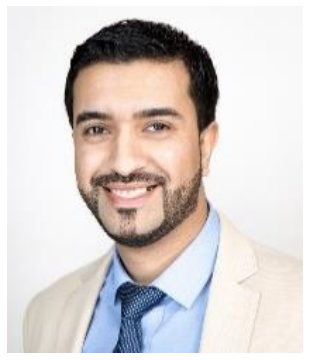

Mohammed Falih Kadhim (D) SD SC P is the directing manager of Software department at ITRDC, University of Kufa. He and his team are responsible for developing and administrating all websites in the University and its' faculties. Earned his bachelor degree in Computer Technique Engineering from the Islamic College University in 2010. Later, he earned his Master degree in Information Technology from the University of Technology, Sydney, Australia in 2015. His research interests are mostly Network and web related such as Front-end Web Development, WSN, Learning Management Systems, Peer-learning using Social Media Platforms. He can be contacted at email: mohammed.kadhim@uokufa.edu.iq.

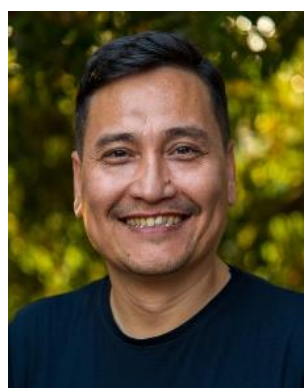

Othman M. Hussein Anssari (iD 8d SC P He received the B. Tech. degree in Control Engineering from the University of Technology, Baghdad, Iraq, 2002. and M. E degree in the Industrial Drive and Control department, University College of Engineering, Osmania University, Hyderabad, India 2015. Technical Support Division Manager at Information Technology Research \& development center, University of Kufa, Al-Najaf, Iraq. He can be contacted at email: othman.alansari@uokufa.edu.iq.

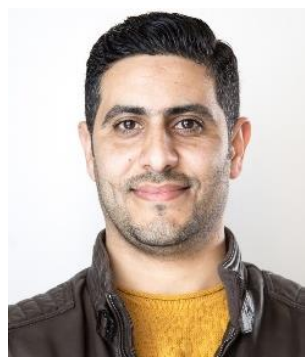

Ahmed AbdulHamza Al-Jobouri (iD S SC P Is a senior engineer currently working at the University of Kufa. He received the B. Tech. degree in Control Engineering from the University of Technology, Baghdad, Iraq, 2006. Currently manage and administrates the e-learning system and Moodle platform for the University of Kufa-Information Technology Research and Development Center, Al-Najaf, Iraq. He can be contacted at email: ahmed.algburi@uokufa.edu.iq. 